\section{J'TI}

JOURNAL OF

TRAUMA AND INJURY

Received: March 16, 2021

Revised: May 29, 2021

Accepted: June 2, 2021

\section{Correspondence to}

Young Hoon Sul, M.D., Ph.D.

Department of Trauma Surgery, Chungbuk National University Hospital,

7761 Sunhwan-ro, Seowon-gu, Cheongju

28644, Korea

Tel: +82-43-269-7847

Fax: +82-43-269-7810

E-mail:ssulyh@gmail.com

ORCID: https://orcid.org/0000-0003-

3184-3396

\title{
Visual Disturbance Caused by a Nail Gun-Induced Penetrating Brain Injury
}

\author{
Jin Bong Ye, M.D. ${ }^{1,2}$, Young Hoon Sul, M.D., Ph.D. ${ }^{1,2,3}$, Se Heon Kim, M.D. ${ }^{1,2}$, \\ Jin Young Lee, M.D. ${ }^{1,2}$, Jin Suk Lee, M.D. ${ }^{1,2}$, Hong Rye Kim, M.D. ${ }^{1,4}$, \\ Soo Young Yoon, M.D. ${ }^{1,5}$, Jung Hee Choi, M.D. ${ }^{1,6}$ \\ ${ }^{1}$ Trauma Center, Chungbuk National University Hospital, Cheongju, Korea \\ ${ }^{2}$ Department of Trauma Surgery, Chungbuk National University Hospital, Cheongju, \\ Korea \\ ${ }^{3}$ Depratment of Trauma Surgery, College of Medicine, Chungbuk National University, \\ Cheongju, Korea \\ ${ }^{4}$ Department of Neurosurgery, Chungbuk National University Hospital, Cheongju, Korea \\ ${ }^{5}$ Department of Thoracic and Cardiovascular Surgery, Chungbuk National University \\ Hospital, Cheongju, Korea \\ ${ }^{6}$ Department of Anesthesiology and Pain Medicine, Chungbuk National University \\ Hospital, Cheongju, Korea
}

Penetrating brain injury caused by a nail gun is an uncommon clinical scenario reported in the literature. A 36-year-old male presented with a nail that had penetrated through the occipital bone. He was alert and neurologically intact except for visual disturbance. Computed tomography (CT) of the brain showed the nail lodged at the occipital lobe and the parietal lobe, with minimal intracerebral hemorrhage. The nail was placed in the occipital lobe close to the superior sagittal sinus. We removed the nail with craniotomy since the entrance of the nail was close to the superior sagittal sinus. There were no newly developed neurological deficits postoperatively. Immediate postoperative CT showed no newly developed lesions. The patient recovered well without any significant complications. Two weeks postoperatively, magnetic resonance imaging showed no remarkable lesions. The visual disturbance was followed up at the outpatient department. To summarize, we report a rare case of penetrating head injury by a nail gun and discuss relevant aspects of the clinical management.

Keywords: Foreign bodies; Head injuries; Visual disturbance; Nail gun 


\section{JTI}

\section{INTRODUCTION}

Non-missile-related penetrating brain injuries (PBIs) have been relatively uncommonly reported in the literature owing to the protective morphology of the skull [1]. Cranial nail gun injuries have been usually associated with mental health-related suicide attempts, instead of industrial nail gun injuries [2].

In this article, we report a case of a patient with an intracranial nail gun injury treated with craniotomy and foreign body removal. The nail was successfully removed with no hemorrhagic or ischemic sequelae.

\section{CASE REPORT}

A 36-year-old, previously healthy male patient who was a foreign construction worker sustained a PBI resulting from the accidental firing of a nail gun on the job. He had not been wearing a safety helmet. The nail hit him in the
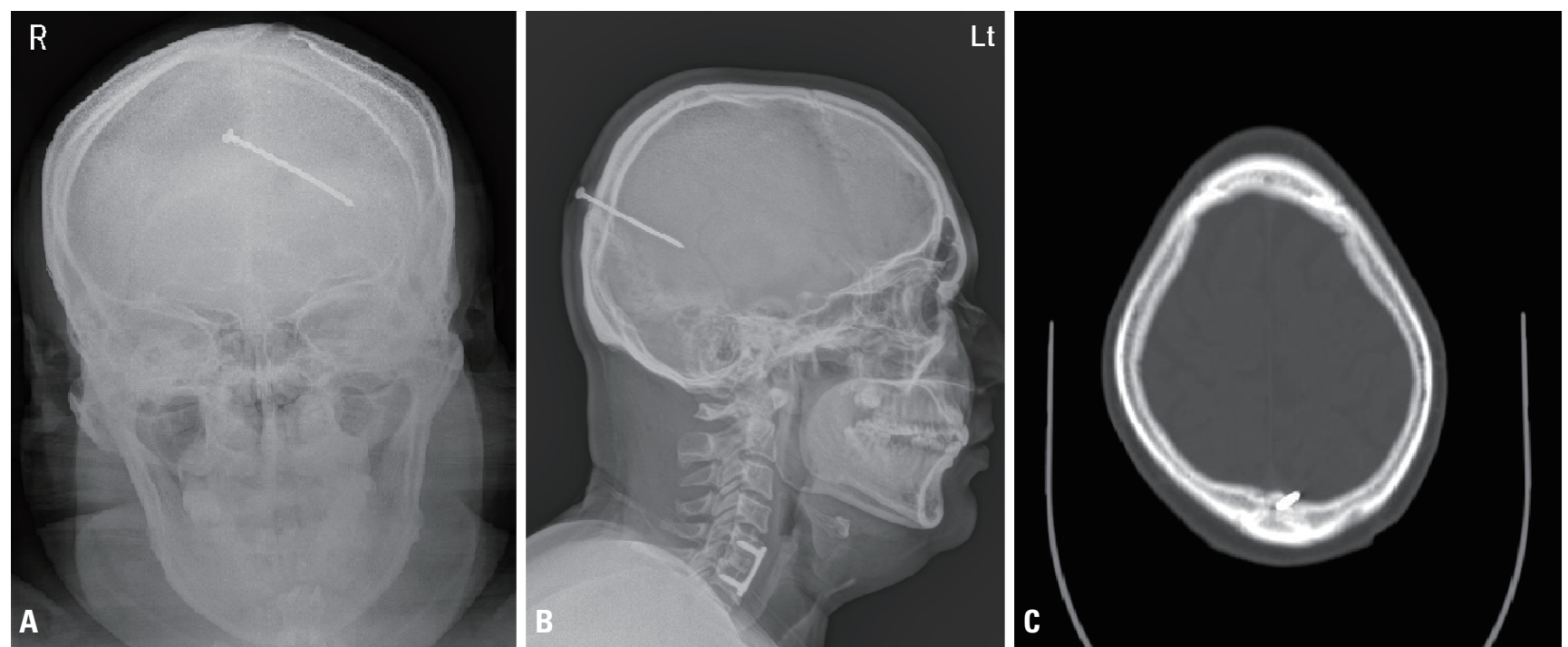

Fig. 1. (A) Plain skull anterior-posterior and (B) lateral views showing the nail placed through the parietal bone near the midline, and (C) axial computed tomography image demonstrating the nail close to the superior sagittal sinus without definite hemorrhage.
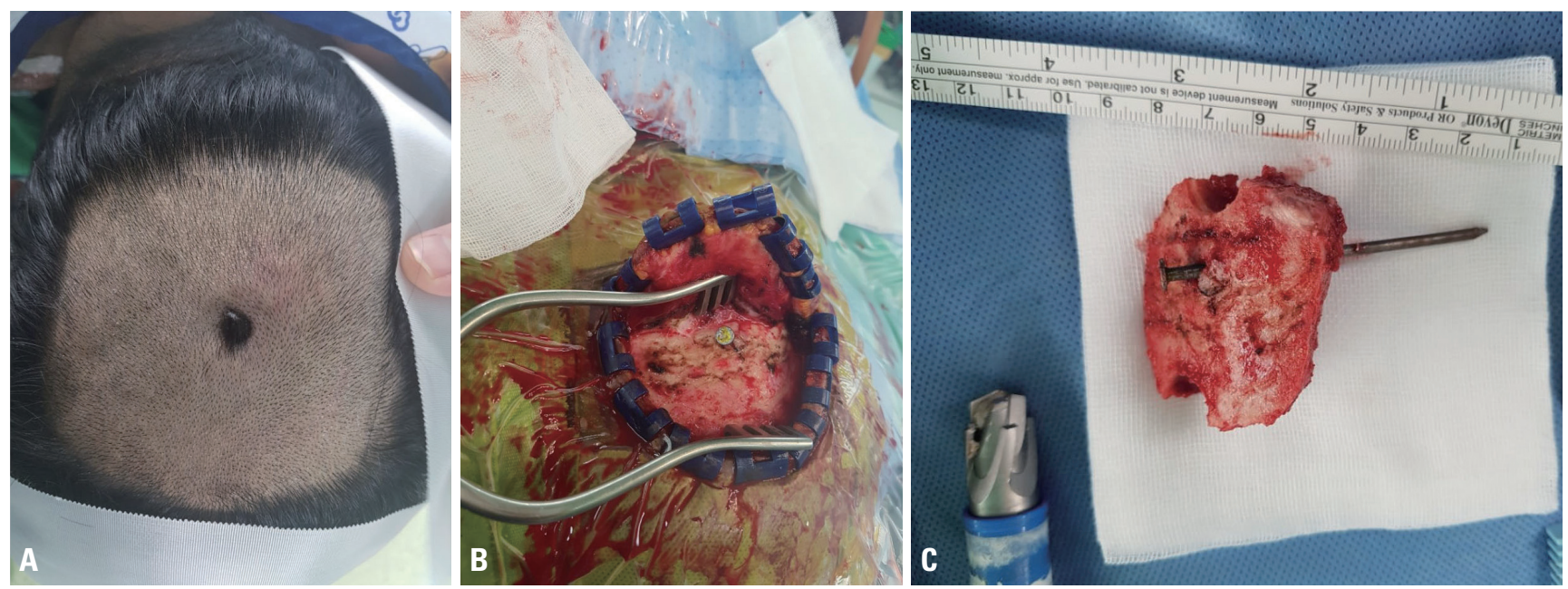

Fig. 2. Intraoperative photography illustrating the nail. (A, B) Surgical field and (C) specimen. 
vertex area.

The patient had no neurological deficit except for a complaint of bilateral blurred near vision upon admission, 3 hours after the accident. On examination, his vital signs were stable. The basic laboratory findings were normal. An ocular inspection of the head after shaving revealed the nail head on the vertex of the skull without hematoma or active signs of bleeding. Skull radiography revealed a $7-\mathrm{cm}$, straight, oblique-oriented, nail-shaped foreign body piercing the brain in the occipital area (Fig. 1). Computed tomography (CT) of the head showed an object of metallic density that had penetrated the skull and entered the brain parenchyma in the occipital region, and the entrance of the nail was close to the sagittal sinus (Fig. 1). However, there was no evidence of major arterial or venous compromise.

The patient was immediately taken for surgery under general anesthesia, and a $15-\mathrm{cm}$ curved skin incision was made across the nail head on both of its sides. A small craniotomy involving the nail penetration site was per- formed. The nail became loose and was pulled out very slowly and gently. After its removal, irrigation of the craniotomy area was performed and routine culture of the nail was done (Fig. 2). The dural defect was repaired. There were no immediate complications after surgery. Postoperatively, the patient was given antibiotics (ceftriaxone [2 $\mathrm{g}$ intravenous daily] and metronidazole [500 mg intravenous every 8 hours]) for 12 days and prophylaxis for tetanus.

Two weeks after the accident, magnetic resonance imaging (MRI) of the brain and visual field testing were performed. A fundoscopic examination revealed normal eye grounds. Visual acuity without correction was 20/60 in both eyes. Visual field testing demonstrated a homonymous pattern of constricted bilateral fields (Fig. 3). MRI showed no remarkable finding except minimal hemorrhage of the injury site, and there were no infection signs (Fig. 4). Visual acuity did not improve during follow-up at the outpatient department. The patient was lost to follow-up after 2 months.
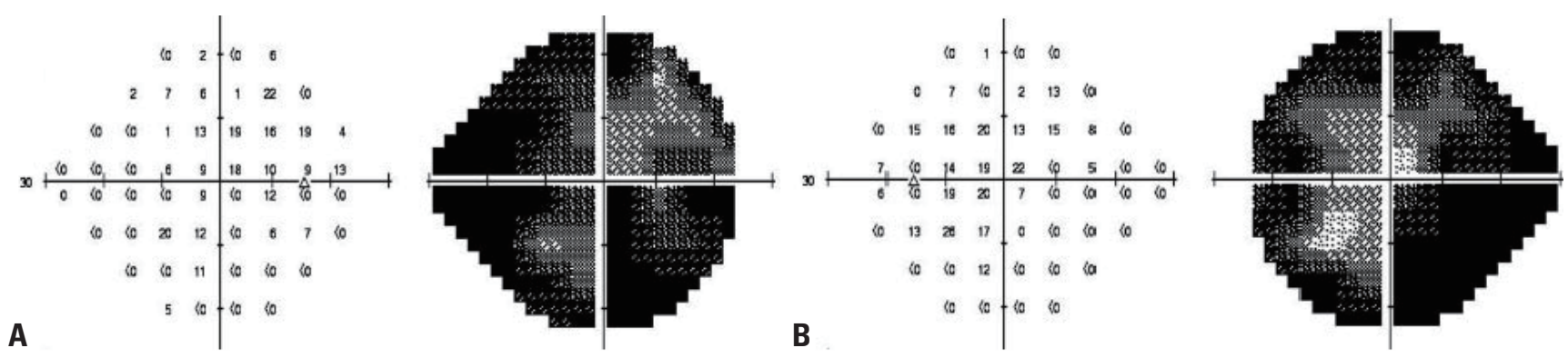

Fig. 3. An automated perimetry printout using a SITA 30-fast to test the central 24-2 threshold test of each eye. (A) Visual field of the right eye and (B) visual field of the left eye.
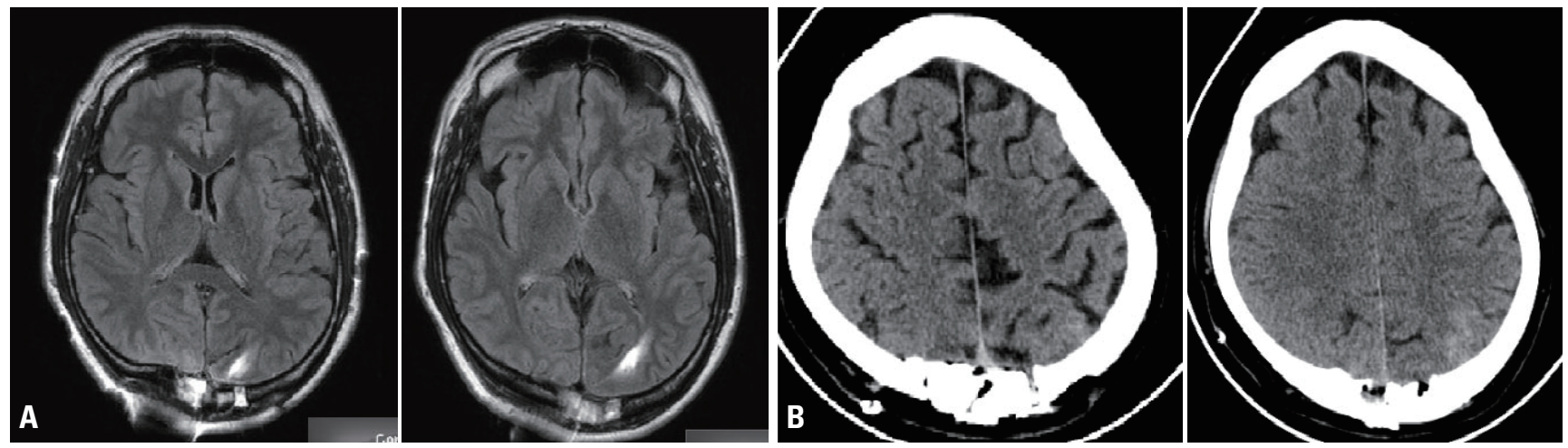

Fig. 4. (A) Postoperative T2 fluid-attenuated inversion recovery magnetic resonance image and (B) postoperative brain computed tomography scan. 


\section{DISCUSSION}

Intracranial nail-gun injuries have more favorable outcomes than penetrating head trauma from firearms due to the relatively low velocity of these projectiles [3]. Although serious nail gun injuries have been reported $[4,5]$, even patients with multiple intracranial nail injuries have been treated with minimal neurological impairment $[6,7]$. Although the intracranial nail was located near the superior sagittal sinus in this case, fortunately no obvious vascular damage or hematoma occurred. Successful treatment of superior sagittal sinus involvement has been reported [8]. Cerebral angiography for evaluating vascular injury is recommended both initially and at follow-up period [9]. Approximately two-thirds of studies in the literature used formal catheter angiography to evaluate vascular abnormalities such as traumatic stenosis, arterial dissection, pseudoaneurysm formation, and vessel penetration [1]. However, in our case, angiography was omitted to minimize the economic burden on a foreign worker without any evidence of vascular injury on CT. The vascular complication rate after PBI ranges from under $5 \%$ to $40 \%$ in various reports [10].

Cerebral angiography is important because a delay in the diagnosis of vascular injury is associated with unfavorable outcomes. Traumatic intracranial pseudoaneurysm is the most commonly described vascular injury after PBI. Cerebral angiography remains the most appropriate modality for diagnosis and treatment [10].

The role of prophylactic antibiotics in PBI has been strongly advocated, but there is no consensus on the efficacy thereof $[10,11]$. In a large retrospective review of civilian PBI data, the rate of infection was documented to be $1-5 \%$ with the use of broad-spectrum antibiotics [11]. Based on the available literature, it is recommended that broad-spectrum antibiotics should be administered in all PBI cases as soon as possible.

The "Infection in Neurosurgery" Working Party of the British Society for Antimicrobial Therapy recommended the following regimen for PBI: intravenous co-amoxiclav (1.2 g, every 8 hours), or intravenous cefuroxime (1.5 g, then $750 \mathrm{mg}$ every 8 hours) with intravenous metronidazole (500 $\mathrm{mg}$, every 8 hours) [12]. It is recommended to start this regimen as soon as possible after the injury and to continue it for 5 days postoperatively.

For patients presenting with brain parenchymal secondary lesions or hemorrhage, antiepileptic drugs (AEDs) should be considered. If seizures are not evident in the acute phase, AEDs may be discontinued after 7 days, but the length of use remains controversial $[13,14]$. It is acceptable not to use prophylactic anticonvulsants at all in case of smaller, less serious trauma. Furthermore, the use of anticonvulsants beyond the first 7 days of injury is generally not recommended $[13,14]$.

All penetrating foreign bodies should be removed under direct vision to treat secondary lesions and to repair the dura mater and prevent cerebrospinal fluid leakage [15]. Spennato et al. [16] described a technique known as double concentric craniotomy, which was commonly performed as a safe and effective treatment modality for intracranial nail injuries. This technique enables removal of the nail under direct vision. In double concentric craniotomy, a small craniotomy is made just around the head of the nail and a larger bone flap, involving the first craniotomy, is performed. The larger bone flap is elevated first, whereas the small bone flap with the nail infixed is carefully elevated along the axis of the nail, under direct vision of the nail tract [16]. However, in this case, ordinary craniotomy was applied for our patient to diminish the risk of superior sagittal sinus injury during the procedure.

Signal changes in the visual cortex in both the occipital lobe and right optic radiations in the parietal lobe were identified in the MRI findings of this case. Optic pathway trauma consisting of damage to the chiasm or the retrochiasmal visual pathways is common in head trauma. $[17,18]$ Chiasmal damage is second to intracanalicular optic nerve segment damage in frequency and may be a result of indirect or penetrating injury. Visual field testing in combination with brain MRI is usually diagnostic; however, little is known about optimal management, and most patients are treated empirically with observation.

PBI by a nail gun is a rare clinical condition. Verification of the type of foreign object and identification of adjacent cerebral vascular structures are important aspects of management to remove the foreign body without further vascular injury or uncontrolled intracranial hemorrhage. This case demonstrated successful surgical management 
of PBI resulting from an industrial nail gun with minimal neurological consequences.

\section{REFERENCES}

1. Woodall MN, Alleyne CH Jr. Nail-gun head trauma: a comprehensive review of the literature. J Trauma Acute Care Surg 2012;73:993-6.

2. Shakir A, Koehler SA, Wecht CH. A review of nail gun suicides and an atypical case report. J Forensic Sci 2003;48:409-13.

3. Centers for Disease Control and Prevention (CDC). Nail-gun injuries treated in emergency departments--United States, 20012005. MMWR Morb Mortal Wkly Rep 2007;56:329-32.

4. Jeon YH, Kim DM, Kim SH, Kim SW. Serious penetrating craniocerebral injury caused by a nail gun. J Korean Neurosurg Soc 2014;56:537-9.

5. Oh HH, Kim Y, Park SC, Ha YS, Lee KC. Nail gun induced open head injury: a case report. Korean J Neurotrauma 2014;10:13941.

6. Litvack ZN, Hunt MA, Weinstein JS, West GA. Self-inflicted nail-gun injury with 12 cranial penetrations and associated cerebral trauma. Case report and review of the literature. J Neurosurg 2006;104:828-34.

7. Makoshi Z, AlKherayf F, Da Silva V, Lesiuk H. Nail gun injuries to the head with minimal neurological consequences: a case series. J Med Case Rep 2016;10:58.

8. Sani S, Jobe KW, Byrne RW. Successful repair of an intracranial nail-gun injury involving the parietal region and the superior sagittal sinus. Case report. J Neurosurg 2005;103:567-9.

9. Blankenship BA, Baxter AB, McKahn GM 2nd. Delayed cerebral artery pseudoaneurysm after nail gun injury. AJR Am J Roentgenol 1999;172:541-2.

10. Kazim SF, Shamim MS, Tahir MZ, Enam SA, Waheed S. Management of penetrating brain injury. J Emerg Trauma Shock 2011;4:395-402.

11. Antibiotic prophylaxis for penetrating brain injury. J Trauma 2001;51(Suppl 2):S34-40.

12. Bayston R, de Louvois J, Brown EM, Johnston RA, Lees P, Pople IK. Use of antibiotics in penetrating craniocerebral injuries. "Infection in neurosurgery" Working Party of British Society for Antimicrobial Chemotherapy. Lancet 2000;355:1813-7.

13. Aarabi B, Taghipour M, Haghnegahdar A, Farokhi M, Mobley L. Prognostic factors in the occurrence of posttraumatic epilepsy after penetrating head injury suffered during military service. Neurosurg Focus 2000;8:e1.

14. Antiseizure prophylaxis for penetrating brain injury. J Trauma 2001;51(Suppl S):S41-3.

15. Awori J, Wilkinson DA, Gemmete JJ, Thompson BG, Chaudhary N, Pandey AS. Penetrating head injury by a nail gun: case report, review of the literature, and management considerations. J Stroke Cerebrovasc Dis 2017;26:e143-9.

16. Spennato P, Bocchetti A, Mirone G, Savarese L, Squillante D, Rotondo M, et al. Double concentric craniotomy for a craniocerebral penetrating nail. Case report and technical note. Surg Neurol 2005;64:368-71; discussion 371.

17. Savino PJ, Glaser JS, Schatz NJ. Traumatic chiasmal syndrome. Neurology 1980;30:963-70.

18. Bruce BB, Zhang X, Kedar S, Newman NJ, Biousse V. Traumatic homonymous hemianopia. J Neurol Neurosurg Psychiatry 2006;77:986-8. 